\title{
Non-commutative space-time of Doubly Special Relativity theories
}

\author{
Jerzy Kowalski-Glikman and Sebastian Nowakf \\ Institute for Theoretical Physics \\ University of Wroctaw \\ Pl. Maxa Borna 9 \\ Pl-50-204 Wroctaw, Poland
}

\begin{abstract}
Doubly Special Relativity (DSR) theory is a recently proposed theory with two observerindependent scales (of velocity and mass), which is to describe a kinematic structure underlining the theory of Quantum Gravity. We observe that there is infinitely many DSR constructions of the energy-momentum sector, each of whose can be promoted to the $\kappa$-Poincaré quantum (Hopf) algebra. Then we use the co-product of this algebra and the known construction of $\kappa$-deformed phase space via Heisenberg double in order to derive the non-commutative space-time structure and description of the whole of the DSR phase space. Next we show that contrary to the ambiguous structure of the energy momentum sector, the space-time of the DSR theory is unique and equivalent to the theory with non-commutative space-time proposed long ago by Snyder. This theory provides non-commutative version of Minkowski space-time enjoying ordinary Lorentz symmetry. It turns out that when one builds a natural phase space on this space-time, its intrinsic length parameter $\ell$ becomes observer-independent.
\end{abstract}

PACS numbers: $02.20 \mathrm{Uw}, 02.40 \mathrm{Gh}, 03.30$

\section{INTRODUCTION}

Most approaches to quantum gravity work in top-tobottom fashion: one tries to define a more or less complete theory (like strings theory or loop quantum gravity) and then to work out some picture of low energy phenomena that can be in principle confronted with experiment. This procedure is in a sharp contrast with the way the General Theory of Relativity has been first formulated. Einstein started with Special Relativity, and then tried to remove the tension between this theory and the Newtonian theory of gravity. Such a bottom-to-top way is certainly easier because one starts with relatively simple problems, and only after getting some experience the more difficult ones are started being addressed. However, contrary to the situation Einstein was facing at the beginning of XX century, on our way to quantum gravity we are in much more difficult situation having in our dis-

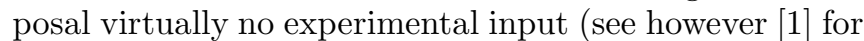
review of the current status of "Quantum Gravity Phenomenology".) The development of the top-to-bottom theories provided us however with some rather robust intuitions as to what the starting point of bottom-to-top procedure should be. First of all quantum gravity should certainly describe the flat geometry, i.e., the structure of space-time when gravitational field is switched off. Second, the top-to-bottom approaches seem to indicate the existence of a fundamental scale, which could be identified with Planck scale (of length or mass.) These two observations form the basis for the studies presented in

\footnotetext{
*Electronic address: jurekk@ift.uni.wroc.p ; Research partially supported by the KBN grant 5PO3B05620.

${ }^{\dagger}$ Electronic address: pantera@ift.uni.wroc.pl
}

this paper.

About a year ago, in his seminal papers [2], [3], [4] G. Amelino-Camelia made the major breakthrough in the bottom-to-top approach to quantum gravity. He observed that if the Planck scale is treated not as a coupling constant, but as an observer-independent scale, then we immediately find ourselves with direct conflict with basic principles of Special Relativity, which teaches us that masses (and lengths) are not observer-independent. To overcome this difficulty Amelino-Camelia proposed to consider theory with two observer-independent scales, dubbed by him the Doubly Special Relativity (DSR). He also suggested that such a theory may have as an algebra of symmetries some kind of a quantum (Hopf) algebra, which would be an extension of the standard Poincaré algebra. The existence of the second observer-independent scale is in this approach a remnant of Quantum Gravity, and thus Doubly Special Relativity is to be viewed therefore as a Quantum Gravity in flat space-time, or, in other words, Quantum Special Relativity.

It turned out [5], [6] that the DSR proposal can be realized in the framework of the quantum $\kappa$-Poincaré algebra [7], [8]. This algebra contains a natural deformation parameter of dimension of mass $\kappa$ by construction (along with the speed of light $c$ which we will put here equal 1.) It has been explicitly showed in [6] that one of the basic physical prediction of the DSR theory with $\kappa$-Poincaré algebra playing the role of an algebra of symmetries is the existence of the fundamental mass scale, which is observer-independent.

The quantum $\kappa$-Poincaré algebra is in the same relation to DSR theory as the standard Poincaré algebra is related to Special Relativity. In other words DSR is a physical theory in which the description of energy-momentum sector is provided by $\kappa$-Poincaré quantum algebra. However the knowledge of this algebra is only the first step 
in construction of the DSR theory. The reason is at least twofold.

First of all the $\kappa$-Poincaré algebra is a quantum nonlinear algebra, and thus, contrary to the standard Poincaré case there are no restrictions on nonlinear transformations among momenta. This means that from the mathematical point of view different bases of the algebra related by arbitrary (analytical) transformations of momenta are completely equivalent. Up to date four such bases have been investigated in details: the "standard" basis with deformed Lorentz sector [7], the bicrossproduct basis [8], the classical basis [11], and recently a new basis proposed by Magueijo and Smolin [12], [13]. It is therefore an open question whether these mathematically equivalent bases are equivalent physically, or if one of them is singled out by some physical considerations.

Secondly, the $\kappa$-Poincaré quantum algebra is an algebra describing in a direct way only the energy-momentum sector of the DSR theory. The knowledge of this sector alone is insufficient to consider many physical problems. Moreover, as argued in 14 if one sticks only to the energy-momentum sector, the resulting theory is based on a brute force, unjustified deformation of the standard Poincaré algebra and thus not very appealing. It is the co-algebra of the quantum algebra that provides a way out of this dilemma. Indeed, as we show below the coalgebra can be used to derive the form of the commutator algebra on the whole of the phase space. The $\kappa$-deformed phase space has been first obtained in [16], 15], 20] for bicrossproduct basis. This result was extended in 13 for other bases. However the emerging picture of the phase space was still unclear. Here we want to investigate the properties of this space in more details. In particular, we will address the question which of these properties are universal, independent of the energy-momentum basis one starts with, and which are not. If indeed all the bases are physically equivalent only the former may correspond to physical "observables".

The plan of this paper is as follows. In sections II and III we show that under rather mild assumption an arbitrary energy-momentum algebra forms, along with the undeformed Lorentz algebra a Hopf algebra. This is a very promising result, since the Hopf algebra structure makes it possible to extend the energy-momentum algebra to the algebra of space-time. This extension turns out to have a remarkable property, namely that the form of space-time non-commutativity is independent of the energy-momentum basis one starts with. This result is proved in section III. Next, in section IV we show that also the way how positions transform under action of Lorentz generators is energy-momentum basis independent. Thus the space-time sector of DSR is perfectly unambiguous, and can therefore serve as a basis to construct a physical theory. Moreover, it turns out that the resulting theory is exactly the one proposed in the paper of Snyder [9], in which the idea of space-time non-commutativity was spelled out for the first time. The natural phase-space algebra associated with Snyder's non-commutative Minkowski space-time is presented in section VI. Section VII is defoted to conclusions and outlooks.

\section{THREE BASES OF $\kappa$-POINCARÉ QUANTUM ALGEBRA}

In this section we present general results describing some algebras furnishing the energy-momentum sector of DSR theory. Our aim here is twofold: first we present concrete examples, of which we will make use in the following sections, and second we prove statements concerning the general nature of $\kappa$-Poincaré quantum algebras, and therefore applicable to any DSR theory.

The $\kappa$-Poincaré quantum algebra is a quantum (Hopf) algebra being a deformation of the Poincaré algebra of special relativity. This algebra has been proposed first in the paper [7] (see also [10] for early review). However the algebra presented there has been written in the so-called standard basis, which does not satisfy the natural requirement that the action of the Lorentz sector integrates to a group. Only few years later in the paper [8] the bicrossproduct basis was introduced in which the Lorentz sector was undeformed. Therefore, to distinguish these two cases we introduce the notion of $\kappa$-DSR algebra which is an $\kappa$-deformed Heisenberg algebra on the phase space of the system with the basis satisfying

1. the Lorentz sector of this algebra is undeformed,

2. the action of rotations on momenta is classical ${ }^{1}$,

3. the space-time commutators and the ones between positions and momenta are uniquely defined by the co-product and appropriate pairing (see below), and

4. in the limit when the deformation parameter $\kappa \rightarrow$ $\infty$ the algebra becomes the classical phase space algebra, i.e, the Poincaré algebra along with the standard canonical commutational relations between positions and momenta (with the trivial co-algebra sector).

To date three examples (or, better to say, bases) of $\kappa$-DSR algebra have been analyzed, and for all of them, according to postulates (a) and (b):

$$
\begin{gathered}
{\left[M_{i}, M_{j}\right]=i \epsilon_{i j k} M_{k}, \quad\left[M_{i}, N_{j}\right]=i \epsilon_{i j k} N_{k},} \\
{\left[N_{i}, N_{j}\right]=-i \epsilon_{i j k} M_{k} .}
\end{gathered}
$$

\footnotetext{
${ }^{1}$ Let us note that the deformation can be associated only with one dimension and it follows from the requirement of rotational symmetry that we choose this direction to be timelike. One should remember however that there exists a different $\kappa$-Poincaré theory in which one deforms the algebra along null direction.
} 
and

$$
\left[M_{i}, p_{j}\right]=i \epsilon_{i j k} p_{k}, \quad\left[M_{i}, p_{0}\right]=0
$$

hold.

\section{1. $\kappa$-Poincaré algebra in the bicrossproduct basis.}

The algebra sector reads

$$
\left[N_{i}, p_{j}\right]=i \delta_{i j}\left(\frac{\kappa}{2}\left(1-e^{-2 p_{0} / \kappa}\right)+\frac{1}{2 \kappa} \vec{p}^{2}\right)-i \frac{1}{\kappa} p_{i} p_{j}
$$

and

$$
\left[N_{i}, p_{0}\right]=i p_{i}
$$

with the first Casimir equal

$$
m^{2}=\left(2 \kappa \sinh \left(\frac{p_{0}}{2 \kappa}\right)\right)^{2}-\vec{p}^{2} e^{p_{0} / \kappa} .
$$

It should be noted in passing the the parameter $m$ above is not the physical mass ${ }^{2}$, in fact, as shown in [13] the correct expression for physical mass has the form

$$
m_{\text {phys }}^{2}=\frac{\kappa^{2}}{4}\left(1-\left(-\frac{m}{2 \kappa}+\sqrt{\frac{m^{2}}{4 \kappa^{2}}+1}\right)^{4}\right)^{2} .
$$

Let us now turn to the co-algebra sector of the $\kappa$ Poincaré algebra. For our present purposes it would be only necessary to know the co-product for the momentum sector. One has

$$
\begin{aligned}
& \Delta\left(p_{i}\right)=p_{i} \otimes \mathbb{1}+e^{-p_{0} / \kappa} \otimes p_{i}, \\
& \Delta\left(p_{0}\right)=p_{0} \otimes \mathbb{1}+\mathbb{1} \otimes p_{0},
\end{aligned}
$$

It is worth noticing that the bicrossproduct basis is singled out by the condition that the energy $p_{0}$ cocommutes.

The co-product is of crucial physical importance, because it makes it possible to construct the space-time sector and the phase space of the theory by a step-by-step procedure. Putting it another way, any construction of the space-time sector is in a sense equivalent to definition of some energy-momentum co-product, and only the one described by eq. (6) has the virtue that together with the commutational relations (11-4) it furnishes a Hopf algebra. It should be stressed at this point that had we not have this structure in our possession, we would not be able to go beyond the energy-momentum sector.

The general procedure of construction of the spacetime commutator algebra from energy-momentum coalgebra consists of the following steps [8], [16]:

\footnotetext{
2 The physical mass is defined by equation $\frac{1}{m_{p h y s}}=\lim _{p \rightarrow 0} \frac{1}{p} \frac{d p_{0}}{d p}$, $p=|\vec{p}|$.
}

1. One defines the bracket (pairing) $<\star, \star>$ between momentum variables $p, q$ and position variables $x, y$ in a natural way as follows

$<p_{\mu}, x_{\nu}>=-i \eta_{\mu \nu}, \quad \eta_{\mu \nu}=\operatorname{diag}(-1,1,1,1)$.

2. This bracket is to be consistent with the co-product structure in the following sense

$$
\begin{aligned}
& <p, x y>=<p_{(1)}, x><p_{(2)}, y>, \\
& <p q, x>=<p, x_{(1)}><q_{(2)}, x_{(2)}>,
\end{aligned}
$$

where we use the natural notation for the coproduct

$$
\Delta t=\sum t_{(1)} \otimes t_{(2)} .
$$

Note that by definition

$$
<\mathbb{1}, 11>=1 .
$$

One sees immediately that the fact that momenta commute translates to the fact that positions cocommute

$$
\Delta x_{\mu}=\mathbb{1} \otimes x_{\mu}+x_{\mu} \otimes \mathbb{1} .
$$

Then the first equation in (8) along with (7) can be used to deduce the form of the space-time commutators.

3. It remains only to derive the commutators (cross relations) between momenta and positions. These can be found from the definition of the so-called Heisenberg double (see [16]) and read

$$
[p, x]=x_{(1)}<p_{(1)}, x_{(2)}>p_{(2)}-x p
$$

where $x p$ above is standard multiplication.

As an example let us apply this procedure in the case of the bicrossproduct basis [8], 16], 15]. It follows from (8) that

$$
<p_{i}, x_{0} x_{j}>=-\frac{1}{\kappa} \delta_{i j}, \quad<p_{i}, x_{j} x_{0}>=0,
$$

from which one gets

$$
\left[x_{0}, x_{i}\right]=-\frac{i}{\kappa} x_{i} .
$$

Using (10) we get the standard relations

$$
\left[p_{0}, x_{0}\right]=i, \quad\left[p_{i}, x_{j}\right]=-i \delta_{i j} .
$$

However it turns out that this algebra contains one more non-vanishing commutator, namely

$$
\left[p_{i}, x_{0}\right]=-\frac{i}{\kappa} p_{i} .
$$


Of course, the algebra (11 13) satisfies the Jacobi identity.

\section{Magueijo-Smolin basis}

In the recent paper 12] Magueijo and Smolin proposed another DSR theory, whose boost generators were constructed as a linear combination of the standard Lorentz generators and the generator of dilatation (but in such a way that the algebra (11) holds.) In this basis the commutators of four-momenta $P_{\mu}$ and boosts have the following form

$$
\left[N_{i}, P_{j}\right]=i\left(\delta_{i j} P_{0}-\frac{1}{\kappa} P_{i} P_{j}\right),
$$

and

$$
\left[N_{i}, P_{0}\right]=i\left(1-\frac{P_{0}}{\kappa}\right) P_{i} .
$$

It is easy to check that the Casimir for this algebra has the form

$$
M^{2}=\frac{P_{0}^{2}-\vec{P}^{2}}{\left(1-\frac{P_{0}}{\kappa}\right)^{2}}
$$

and that $M$ above is the physical mass.

One easily checks that the relation between variables $P_{\mu}$ and $p_{\mu}$ of bicrossproduct basis is given by

$$
\begin{gathered}
p_{i}=P_{i} \\
p_{0}=-\frac{\kappa}{2} \log \left(1-\frac{2 P_{0}}{\kappa}+\frac{\vec{P}^{2}}{\kappa^{2}}\right), \\
P_{0}=\frac{\kappa}{2}\left(1-e^{-2 p_{0} / \kappa}+\frac{\vec{p}^{2}}{\kappa^{2}}\right) .
\end{gathered}
$$

Using formulas above one can without difficulty promote this algebra to the quantum algebra. This amounts only in using the homomorphisms (17), (18) to define the new co-products. They read

$$
\begin{gathered}
\triangle\left(P_{i}\right)=P_{i} \otimes 1+\left(1-\frac{2 P_{0}}{\kappa}+\frac{\vec{P}^{2}}{\kappa^{2}}\right)^{1 / 2} \otimes P_{i} \\
\triangle\left(P_{0}\right)=P_{0} \otimes 1+1 \otimes P_{0}-\frac{2}{\kappa} P_{0} \otimes P_{0}+\frac{1}{\kappa^{2}} \vec{P}^{2} \otimes P_{0}+ \\
+\frac{1}{\kappa}\left(1-\frac{2 P_{0}}{\kappa}+\frac{\vec{P}^{2}}{\kappa^{2}}\right)^{1 / 2} \sum P_{i} \otimes P_{i}
\end{gathered}
$$

To find the non-commutative structure of space time in Magueijo-Smolin basis we start again with eq. (7)

$$
<P_{\mu}, X_{\nu}>=-i \eta_{\mu \nu}
$$

Let us now turn to the next step, eq. (8). It is easy to see that the only terms in (19), 20), which are relevant for our computations are the bilinear ones, so we can write

$$
\begin{gathered}
\triangle\left(P_{i}\right)=\mathbb{1} \otimes P_{i}+P_{i} \otimes \mathbb{1}-\frac{1}{\kappa} P_{0} \otimes P_{i}+\ldots \\
\triangle\left(P_{0}\right)=\mathbb{1} \otimes P_{0}+P_{0} \otimes \mathbb{1}-\frac{2}{\kappa} P_{0} \otimes P_{0}+\frac{1}{\kappa} \sum P_{i} \otimes P_{i}+\ldots
\end{gathered}
$$

It follows immediately that the only non-vanishing commutators in the position sector are

$$
\left[X_{0}, X_{i}\right]=-\frac{i}{\kappa} X_{i}
$$

Now we can use eq. (10) to derive the form of the remaining commutators. Since this computation is a bit tricky, let us present the necessary steps.

$$
\begin{gathered}
{\left[P_{0}, X_{i}\right]=\sum_{j}\left\langle\frac{1}{\kappa} \sqrt{1-\frac{2 P_{0}}{\kappa}+\frac{\vec{P}^{2}}{\kappa^{2}}} P_{j}, X_{i}\right\rangle P_{j}+} \\
+X_{i}<1,11>P_{0}-X_{i} P_{0}= \\
=\frac{1}{\kappa} \sum_{j}<P_{j}, X_{i}>P_{j}
\end{gathered}
$$

(we made use of the fact that the only terms linear in momenta have non-vanishing bracket with positions) from which it follows immediately that

$$
\left[P_{0}, X_{i}\right]=-\frac{i}{\kappa} P_{i}
$$

and by employing the same procedure we obtain the remaining commutators

$$
\begin{gathered}
{\left[P_{0}, X_{0}\right]=i\left(1-\frac{2 P_{0}}{\kappa}\right)} \\
{\left[P_{i}, X_{j}\right]=-i \delta_{i j}} \\
{\left[P_{i}, X_{0}\right]=-\frac{i}{\kappa} P_{i} .}
\end{gathered}
$$

Of course, as it is easy to check, the algebra above satisfies the Jacobi identity.

Let us note in passing an interesting property of the commutator (23) namely that for states with energy $\kappa / 2$ $P_{0}$ and $X_{0}$ commute. However since it is our view that one should not treat any particular energy-momentum algebra as a physical one unless there are appealing physical arguments to think otherwise, we will not dwell on this observation. 


\section{The classical basis}

There is yet another basis which we will present here for comparison (this basis was first described in 17; see also [11], [18], [19].) In this basis, which we call the classical one, the boosts-momenta commutators together with the Lorentz sector form the classical Poincaré algebra, to wit

$$
\left[N_{i}, \mathcal{P}_{j}\right]=i \delta_{i j} \mathcal{P}_{0}, \quad\left[N_{i}, \mathcal{P}_{0}\right]=i \mathcal{P}_{i}
$$

The Casimir for this basis equals, of course the one of special relativity, to wit

$$
\mathcal{M}^{2}=\mathcal{P}_{0}^{2}-\overrightarrow{\mathcal{P}}^{2}
$$

The classical generators $\mathcal{P}_{\mu}$ are related to the bicrossproduct basis generators by the formulas

$$
\begin{gathered}
\mathcal{P}_{0}=\kappa \sinh \frac{p_{0}}{\kappa}+e^{p_{0} / \kappa} \frac{\vec{p}^{2}}{2 \kappa}, \\
\mathcal{P}_{i}=e^{p_{0} / \kappa} p_{i}
\end{gathered}
$$

and one can easily compute the expression for co-product

$$
\begin{gathered}
\Delta\left(\mathcal{P}_{0}\right)=\frac{\kappa}{2}\left(K \otimes K-K^{-1} \otimes K^{-1}\right)+ \\
+\frac{1}{2 \kappa}\left(K^{-1} \overrightarrow{\mathcal{P}}^{2} \otimes K+2 K^{-1} \mathcal{P}_{i} \otimes \mathcal{P}_{i}+K^{-1} \otimes K^{-1} \overrightarrow{\mathcal{P}}^{2}\right) \\
\Delta\left(\mathcal{P}_{i}\right)=\mathcal{P}_{i} \otimes K+11 \otimes \mathcal{P}_{i}
\end{gathered}
$$

where

$$
K=e^{p_{0} / \kappa}=\frac{1}{\kappa}\left[\mathcal{P}_{0}+\left(\mathcal{P}_{0}^{2}-\overrightarrow{\mathcal{P}}^{2}+\kappa^{2}\right)^{1 / 2}\right] .
$$

To derive the phase space commutators, we again start with the duality relation

$$
<\mathcal{P}_{\mu}, \mathcal{X}_{\nu}>=-i \eta_{\mu \nu}
$$

and to get the commutators in the position sector as above we take the part of the co-product up to the bilinear terms

$$
\begin{gathered}
\triangle\left(\mathcal{P}_{i}\right)=\mathbb{1} \otimes \mathcal{P}_{i}+\mathcal{P}_{i} \otimes \mathbb{1}+\frac{1}{\kappa} \mathcal{P}_{i} \otimes \mathcal{P}_{0}+\ldots \\
\triangle\left(\mathcal{P}_{0}\right)=\mathbb{1} \otimes \mathcal{P}_{0}+\mathcal{P}_{0} \otimes \mathbb{1}+\frac{1}{\kappa} \sum \mathcal{P}_{i} \otimes \mathcal{P}_{i}+\ldots
\end{gathered}
$$

which leads again to

$$
\left[\mathcal{X}_{0}, \mathcal{X}_{i}\right]=-\frac{i}{\kappa} \mathcal{X}_{i}
$$

Then by employing the same method as in the preceding subsection we find the space-time commutators

$$
\begin{gathered}
{\left[\mathcal{P}_{0}, \mathcal{X}_{0}\right]=\frac{i}{2}\left(K+K^{-1}-\frac{1}{\kappa^{2}} \overrightarrow{\mathcal{P}}^{2} K^{-1}\right)} \\
{\left[\mathcal{P}_{0}, \mathcal{X}_{i}\right]=-\frac{i}{\kappa} \mathcal{P}_{i}} \\
{\left[\mathcal{P}_{i}, \mathcal{X}_{j}\right]=-i K \delta_{i j}} \\
{\left[\mathcal{P}_{i}, \mathcal{X}_{0}\right]=0}
\end{gathered}
$$

From these three examples of $\kappa$-DSR algebras, we see that the number of different algebras of this type is unlimited. Even worse, if we are to treat them seriously, we see that they differ by fundamental physical predictions like the existence of maximal momentum and/or energy, the form of dispersion relation etc. They share however, quite unexpectedly, one feature, namely the form of space-time non-commutativity. Keeping this in mind, let us now turn to considerations of more general character.

\section{GENERAL $\kappa$-DSR ALGEBRA}

Consider a generic algebra satisfying the conditions (a)-(d) above. The Lorentz sector of such algebra is given by eq. (11). The most general expression for commutators of boosts and momenta, compatible with rotational symmetry takes the form

$$
\left[N_{i}, p_{j}\right]=\delta_{i j} A+p_{i} p_{j} B+\epsilon_{i j k} p_{k} D
$$

$$
\left[N_{i}, p_{0}\right]=C p_{i}
$$

where $A, B, C, D$ are functions of $p_{0}$ and $\vec{p}^{2}$ only and in the limit $\kappa \rightarrow \infty, A$ and $C$ go to 1 and $B$ and $D$ go to 0 . One easily sees that the Jacobi identity for the commutator (40) forces the function $D=0$, and the one for (39) gives the following condition for the remaining three functions

$$
\frac{\partial A}{\partial p_{0}} C+2 \frac{\partial A}{\partial \vec{p}^{2}}\left(A+\vec{p}^{2} B\right)-A B=1 .
$$

This condition can be solved for example for $B$ and we see that only two of the functions $A, B, C$ are independent. Recalling now that the most general transformation from the classical basis to another one satisfying the condition of rotational covariance has the form

$$
p_{i}=\alpha\left(\mathcal{P}_{0}, \overrightarrow{\mathcal{P}}^{2}\right) \mathcal{P}_{i}, \quad p_{0}=\beta\left(\mathcal{P}_{0}, \overrightarrow{\mathcal{P}}^{2}\right),
$$

and is defined by two arbitrary functions $\alpha$ and $\beta$, and that, after substituting to eqs. (26) one obtains equations 
of the form (39), (40), we see that any DSR basis can be obtained from the classical basis in this way.

One should remember however that the functions $\alpha$ and $\beta$ are not completely arbitrary. The important physical requirement is that any basis has the standard Poincaré algebra as its limit at $\kappa \rightarrow \infty$. This means that, for large $\kappa$ we have

$$
\begin{gathered}
\alpha\left(\mathcal{P}_{0}, \overrightarrow{\mathcal{P}}^{2}\right) \sim 1+a \frac{\mathcal{P}_{0}}{\kappa}+O\left(\frac{1}{\kappa^{2}}\right), \\
\beta\left(\mathcal{P}_{0}, \overrightarrow{\mathcal{P}}^{2}\right) \sim \mathcal{P}_{0}\left(1+b \frac{\mathcal{P}_{0}}{\kappa}+O\left(\frac{1}{\kappa^{2}}\right)\right),
\end{gathered}
$$

where $a, b$ are numerical parameters, from which it follows that

$$
\begin{aligned}
& \mathcal{P}_{i} \sim\left(1-a \frac{p_{0}}{\kappa}+O\left(\frac{1}{\kappa^{2}}\right)\right) p_{i}, \\
& \mathcal{P}_{0} \sim\left(1-b \frac{p_{0}}{\kappa}+O\left(\frac{1}{\kappa^{2}}\right)\right) p_{0} .
\end{aligned}
$$

This observation has important consequences. To see why, let us consider the commutator $\left[x_{0}, x_{i}\right]$ in the basis defined by eqs. (42). The first step is to find the form of the co-product for $p_{i}, p_{0}$ in the new basis. Using eqs. (32), (33), and (44) we have

$$
\begin{gathered}
\Delta\left(p_{i}\right)=\mathbb{1} \otimes p_{i}+p_{i} \otimes \mathbb{1}+\frac{a}{\kappa} p_{0} \otimes p_{i}+\frac{1+a}{\kappa} p_{i} \otimes p_{0}+\ldots \\
\Delta\left(p_{0}\right)=\mathbb{1} \otimes p_{0}+p_{0} \otimes \mathbb{1}+\frac{1}{\kappa} \sum p_{i} \otimes p_{i}+\ldots
\end{gathered}
$$

where ... denote terms that do not contribute to the commutator. Following our prescription of the preceding section we find that

$$
<p_{i}, x_{0} x_{j}>=\frac{a}{\kappa} \delta_{i j}, \quad<p_{i}, x_{j} x_{0}>=\frac{1+a}{\kappa} \delta_{i j},
$$

from which one gets

$$
\left[x_{0}, x_{i}\right]=-\frac{i}{\kappa} x_{i} .
$$

This shows that the form of space-time noncommutativity is a basis-independent property of any $\kappa$ DSR algebra.

\section{SPACE-TIME LORENTZ TRANSFORMATIONS}

Having obtained the general form of $\kappa$-DSR algebra, let us turn to the next problem. In this section we derive the transformation laws that govern the action of boosts on space-time variables. There are two equivalent ways to find this. One can make use of the Jacobi identity for the algebra consisting of Lorentz generators, commutators of boosts with momenta and the phase space algebra to derive the commutators $\left[N_{i}, x_{\mu}\right]$. In other words one assumes that the phase space commutators transform covariantly under boosts. There is another way to get the same result, however. To do that one takes the co-product for boosts and proceeds following exactly the same steps as the ones that led us to phase space algebra. In this way the Jacobi identity for the whole of the algebra (including the commutators of boosts with space time variables) is guaranteed by construction.

Let us start with co-product for the boosts in the bicrossproduct basis

$$
\Delta\left(N_{i}\right)=N_{i} \otimes 1+e^{-p_{0} / \kappa} \otimes N_{i}+\frac{1}{\kappa} \epsilon_{i j k} p_{j} \otimes M_{k}
$$

along with the natural definition for pairing

$$
<N_{i}, x_{j}>=i \delta_{i j} x_{0}, \quad<N_{i}, x_{0}>=i x_{i} .
$$

Then, after simple computations, which follow the steps leading to eq. (12), (13) we get 15], 20]

$$
\left[N_{i}, x_{j}\right]=i \delta_{i j} x_{0}-\frac{i}{\kappa} \epsilon_{i j k} M_{k}, \quad\left[N_{i}, x_{0}\right]=i x_{i}-\frac{i}{\kappa} N_{i} .
$$

One can easily check that this algebra together with (14), (15), (11-13) satisfies Jacobi identity. One can write the commutators (48) in a slightly more convenient way by using the following representation for rotation and boost generators

$$
M_{i}=\epsilon_{i j k} x_{j} p_{k}, \quad N_{i}=x_{i}\left(\frac{\kappa}{2}\left(1-e^{-2 p_{0} / \kappa}\right)+\frac{\vec{p}^{2}}{2 \kappa}\right)-x_{0} p_{i},
$$

to get

$$
\begin{gathered}
{\left[N_{i}, x_{j}\right]=i \delta_{i j} x_{0}-\frac{i}{\kappa} x_{i} p_{j}+\frac{i}{\kappa} x_{j} p_{i}} \\
{\left[N_{i}, x_{0}\right]=i x_{i}\left(\frac{1}{2}\left(1+e^{-2 p_{0} / \kappa}\right)-\frac{\vec{p}^{2}}{2 \kappa^{2}}\right)+\frac{i}{\kappa} x_{0} p_{i} .}
\end{gathered}
$$

It is easy to see that the expression (48) is basis independent. To check this, consider any basis $\left(P_{0}, P_{i}, X_{0}, X_{i}\right)$ related to the bicrossproduct one by

$$
p_{i}=\alpha\left(P_{0}, \vec{P}^{2}\right) P_{i}, \quad p_{0}=\beta\left(P_{0}, \vec{P}^{2}\right),
$$

where the functions $\alpha$ and $\beta$ again have the expansion

$$
\begin{gathered}
\alpha\left(P_{0}, \vec{P}^{2}\right) \sim 1+a \frac{P_{0}}{\kappa}+O\left(\frac{1}{\kappa^{2}}\right), \\
\beta\left(P_{0}, \vec{P}^{2}\right) \sim P_{0}\left(1+b \frac{P_{0}}{\kappa}+O\left(\frac{1}{\kappa^{2}}\right)\right) .
\end{gathered}
$$


Taking now the pairing as above,

$$
\begin{gathered}
<N_{i}, X_{j}>=i \delta_{i j} X_{0}, \quad<N_{i}, X_{0}>=i X_{i}, \\
<P_{\mu}, X_{\nu}>=-i \eta_{\mu \nu}
\end{gathered}
$$

we get

$$
\begin{gathered}
{\left[N_{i}, X_{j}\right]=} \\
\mathbb{1}<N_{i}, X_{j}>\mathbb{1}+\frac{1}{\kappa} \epsilon_{i k l}<\alpha\left(P_{0}, \vec{P}^{2}\right) P_{k}, X_{j}>M_{l}= \\
=i \delta_{i j} X_{0}-\frac{i}{\kappa} \epsilon_{i j k} M_{k} .
\end{gathered}
$$

Similarly

$$
\begin{aligned}
{\left[N_{i}, X_{0}\right]=\mathbb{1}<N_{i}, X_{0} } & >\mathbb{1}+\mathbb{1}<e^{-\beta\left(P_{0}, \vec{P}^{2}\right) / \kappa}, X_{0}>N_{i}= \\
& =i X_{i}-\frac{i}{\kappa} N_{i} .
\end{aligned}
$$

Let us note that by using an appropriate form of $M_{i}, N_{i}$ generators expressed in terms of $X_{\mu}, P_{\mu}$ one can obtain the result similar to (49) in any basis.

The next natural question to be asked is what is a natural form, which replaces the Minkowski metric. To find it let us observe that by replacing $X_{i}$ with

$$
\tilde{X}_{i}=X_{i}-\frac{1}{\kappa} N_{i},
$$

the algebra (51), (52) takes the simple form

$$
\left[N_{i}, \tilde{X}_{j}\right]=i X_{0}, \quad\left[N_{i}, X_{0}\right]=i \tilde{X}_{j},
$$

from which it follows that the invariant quadratic form is

$$
X_{0}^{2}-\tilde{X}_{i} \tilde{X}_{i}=X_{0}^{2}-\left(X_{i}-\frac{1}{\kappa} N_{i}\right)\left(X_{i}-\frac{1}{\kappa} N_{i}\right) .
$$

This quadratic form is again universal, and invariant for any basis.

\section{INTERMEZZO: SO WHERE ARE WE?}

Let us pause for a moment to summarize what we have achieved so far. We started from a list of energymomentum quantum algebras and by making use of their co-product structure we derived the basis-independent space-time commutators. By using the same method we found that the form of commutator of boosts with positions is also basis-independent. This means that the whole space-time sector of $\kappa$-DSR is unambiguous. Instead of using the $X_{i}$ position variables following from the Heisenberg double procedure [15], [20], we find it more convenient to use the combination $\tilde{X}_{i}$ defined by (53). In terms of these variables space-time of DSR is described by the following commutators (because we are now in the space-time sector, it is reasonable to replace the deformation parameter $\kappa$ with the parameter $\ell$ of dimension of length.)

$$
\begin{gathered}
{\left[M_{i}, M_{j}\right]=i \epsilon_{i j k} M_{k}, \quad\left[M_{i}, N_{j}\right]=i \epsilon_{i j k} N_{k},} \\
{\left[N_{i}, N_{j}\right]=-i \epsilon_{i j k} M_{k} .} \\
{\left[X_{0}, \tilde{X}_{i}\right]=-i \ell^{2} N_{i}, \quad\left[\tilde{X}_{i}, \tilde{X}_{j}\right]=i \ell^{2} \epsilon_{i j k} M_{k} .} \\
{\left[N_{i}, \tilde{X}_{j}\right]=i X_{0}, \quad\left[N_{i}, X_{0}\right]=i \tilde{X}_{j},} \\
{\left[M_{i}, \tilde{X}_{j}\right]=i \epsilon_{i j k} \tilde{X}_{k}, \quad\left[M_{i}, X_{0}\right]=0,}
\end{gathered}
$$

It is a remarkable fact that this algebra provides the description of non-commutative Minkowski space (cf., the invariant element (55)) enjoying ordinary Lorentz symmetry, and is identical with the algebra postulated by Snyder in his seminal paper [9], where the idea of spacetime non-commutativity was contemplated for the first time. From our perspective this algebra is exactly the universal basis of the Doubly Special Relativity theory, we have been looking for, and thus this is the algebra (56 59) which is to be taken as a starting point in construction of the DSR theory and analysis of its physical predictions. Specifically, this algebra furnishes the structure of configuration space of the DSR and thus can be used to construct the second order particle lagrangian. This in turn will make it possible to define physical fourmomenta defined by particle dynamics and not the formal algebraic manipulations. However, it is still of interest to investigate the natural phase space basis associated with the space-time algebra above.

\section{THE SNYDER'S BASIS}

Promoting (56 58) to the universal space-time algebra brings the problem of construction of Doubly Special Relativity into completely new perspective. In short we would like to find a representation of this algebra on the space of functions of four commuting variables, which can be identified with components of momenta. There are, of course many such representations, which correspond to different energy momentum bases discussed above. However there is clearly the most natural basis, namely the one considered by Snyder. In this basis the Lorentz generators are given by classical formulas, to wit

$$
M_{i}=\epsilon_{i j k} \tilde{X}_{j} P_{k}, \quad N_{i}=\left(\tilde{X}_{i} P_{0}-X_{0} P_{i}\right)
$$

from which one can derive the form of the commutators $\left[P_{\mu}, X_{\nu}\right]$ as follows.

$$
\left[P_{i}, \tilde{X}_{j}\right]=-i \delta_{i j}-i \ell^{2} P_{i} P_{j},
$$




$$
\begin{gathered}
{\left[P_{0}, X_{0}\right]=i\left(1-\ell^{2} P_{0}^{2}\right),} \\
{\left[P_{i}, X_{0}\right]=-\left[\tilde{X}_{i}, P_{0}\right]=-i \ell^{2} P_{i} P_{0} .}
\end{gathered}
$$

It can be easily checked that the variables $P_{\mu}$ transform under rotations and boosts in a classical way, to wit

$$
\begin{aligned}
& {\left[M_{i}, P_{j}\right]=i \epsilon_{i j k} P_{k}, \quad\left[M_{i}, P_{0}\right]=0,} \\
& {\left[N_{i}, P_{j}\right]=i \delta_{i j} P_{0}, \quad\left[N_{i}, P_{0}\right]=i P_{i} .}
\end{aligned}
$$

Thus the Snyder's basis is a different, and certainly more convenient realization of a basis with classical energymomentum algebra. Indeed, returning to the generators $X_{i}=\tilde{X}_{i}+\ell N_{i}$ we find the relations

$$
\begin{gathered}
{\left[P_{i}, X_{j}\right]=-i \delta_{i j}\left(1+\ell P_{0}\right)-i \ell^{2} P_{i} P_{j},} \\
{\left[X_{i}, P_{0}\right]=i \ell P_{i}+i \ell^{2} P_{i} P_{0},}
\end{gathered}
$$

as compared with the non-polynomial relations $35-38$. Of course, one can work out the co-product structure for the algebra (64), (65) resulting in relations (66), (67)

It is a remarkable fact that the algebra (61) once reduced to one space dimension is exactly the one considered by many as an example of deformed commutational relations leading to generalized uncertainty relations indicating existence of the minimal length. The detailed discussion of quantum mechanical structure underlying this uncertainty relation can be found in [21] and we will not repeat it here. One should note however that the extension to three dimension presented in this paper differs from ours.

We see therefore that the algebra (60-63) predicts the existence of minimal length equal $\ell$. The question arises as to if this minimal length is observer-independent, as required by DSR (the speed o light is of course observerindependent because the dispersion relation is classical.) By this we mean the following. Suppose we have two inertial observers attempting do find out what is their value of the minimal length associated with their generalized uncertainty relation. For both of them this minimal length is associated with the value of the parameter $\ell$ in their commutational relations. The statement that the minimal length is observer-independent is equivalent therefore with the statement that the parameter $\ell$ is the same in any inertial frame. To see that this is indeed the case let us consider the infinitesimal boosts parameterized by parameter $\varepsilon^{k}$

$$
\tilde{X}_{i}^{\prime}=\tilde{X}_{i}+\frac{1}{i} \varepsilon^{k}\left[N_{k}, \tilde{X}_{i}\right], \quad P_{i}^{\prime}=P_{i}+\frac{1}{i} \varepsilon^{k}\left[N_{k}, P_{i}\right]
$$

and consider the commutator $\left[P_{i}^{\prime}, \tilde{X}_{j}^{\prime}\right]$ to order $\varepsilon$. One can easily check that to this order

$$
\left[P_{i}^{\prime}, \tilde{X}_{j}^{\prime}\right]=-i \delta_{i j}-i \ell^{2} P_{i}^{\prime} P_{j}^{\prime},
$$

which is the desired result. It should be stressed that to get this result one should make use of eq. (63) in a nontrivial way.

\section{CONCLUSIONS AND OUTLOOK}

Let us conclude this paper with an outline of the logic of derivations presented above. We started with a simple observation that if one wants to base the DSR theory on the energy-momentum sector, one encounters immediately the problem as to which of mathematically equivalent bases of $\kappa$-Poincaré algebra should be taken as a starting point. There are clearly two possibilities: either one of the bases is distinguished or one should look for some basis independent elements of the DSR theory. The next observation is that the sole energy-momentum sector is insufficient to construct a physical theory; one needs the whole phase space.

At this point the quantum structure of $\kappa$-Poincaré algebra turns out to be crucial. It is only for existence of co-product that one can construct the space-time sector is an unambiguous way. It should be stressed at this point that this provides the physical interpretation of coproduct in the case of algebra of space-time symmetries. Thus the co-product of momenta is the tool enabling the construction of space-time.

Having constructed the phase space, we observe that the structure of space-time sector, i.e., the space-time non-commutativity as well as the action of boosts and rotations on space-time variables is actually completely independent of the energy-momentum basis we started with. We have therefore a non-commutative space-time, which might be, like space-time of Special Relativity, an arena for physical phenomena. It seems reasonable to take this space-time structure as a starting point of investigation of the DSR theory. One should stress at this point that the uniqueness is not the only virtue of the space-time algebra derived in this paper. First of all, when the space-time non-commutativity is expressed in terms of variables $\tilde{X}_{i}$ on obtains the algebra (57) being the simplest possible extension of the non-commutative algebra motivated by string theory (for recent review see 22])

$$
\left[X_{\mu}, X_{\nu}\right]=i \Theta_{\mu \nu}
$$

where $\Theta_{\mu \nu}$ is central element, which (contrary to (69)) manifestly preserves Lorentz invariance. Second if following Snyder [9] one takes a natural representation of the space-time algebra one discovers the natural appearance of observer-independent length scale $\ell$. At this point we would decline from any strong statement as to if this particular phase space basis is physical, i.e., if the variables $P_{\mu}$ of Snyder's basis are to be identified with physical momenta, though certainly this basis looks very promising in this respect. Third the construction presented above provides a non-commutative Minkowski space with undeformed Lorentz symmetry.

There is of course a lot of open problems and possible areas of future research. It seems to us that the most urgent one is to try to construct a natural particle dynamics on non-commutative space-time. This would enable one to identify physical momentum associated with 
particle motion. Another approach to the same problem would be to formulate a field theory on our noncommutative space-time along the lines proposed long ago in 23]. Quantization of such theory would also most likely make it possible to find out what the physical momentum is. Last but not least the fact that along with a unique space-time we seem to have plethora of energymomentum spaces certainly requires explanation.

\section{Acknowledgement}

We would like to thank J. Lukierski for discussions and comments on early version of the manuscript.
[1] G. Amelino-Camelia, gr-qc/0204051 and references therein.

[2] G. Amelino-Camelia, Int. J. Mod. Phys. D 11, 35 (2002), $\mathrm{gr}-\mathrm{qc} / 0012051$.

[3] G. Amelino-Camelia, Phys. Lett. B 510, 255, (2001), hep-th/0012238.

[4] G. Amelino-Camelia, gr-qc/0106004, Proceedings of the 37th Karpacz Winter School on Theoretical Physics, to appear.

[5] J. Kowalski-Glikman, Phys. Lett. A 286, 391 (2001), hep-th/0102098.

[6] N.R. Bruno, G. Amelino-Camelia, and J. KowalskiGlikman, Phys. Lett. B 522, 133 (2001), hep-th/0107039.

[7] J. Lukierski, A. Nowicki, H. Ruegg and V.N. Tolstoy, Phys. Lett. B264, 331 (1991).

[8] S. Majid and H. Ruegg, Phys. Lett. B334, 348 (1994).

[9] H.S. Snyder, Phys. Rev. 71, 38 (1947).

[10] H. Ruegg in Integrable Systems, Quantum Groups, and Quantum Field Theories, L.A. Ibort and M.A. Rodriguez eds., NATO ASI series, vol. 409, Kluwer Academic Publishers, Dordrecht, 1993; J. Lukierski, H. Ruegg and V.N. Tolstoy, in Quantum Groups: Formalism and Applications, Polish Scientific Publishers, (1995), p. 259.

[11] P. Kosiński, J. Lukierski, P. Maslanka, J. Sobczyk, Mod. Phys. Lett. A10, 2599 (1995).

[12] J. Magueijo and L. Smolin, hep-th/0112090.
[13] J. Kowalski-Glikman and S. Nowak, hep-th/0203040

[14] J. Lukierski and A. Nowicki, hep-th/0203065.

[15] J. Lukierski and A. Nowicki, Proceedings of Quantum Group Symposium at Group 21, (July 1996, Goslar) Eds. H.-D. Doebner and V.K. Dobrev, Heron Press, Sofia, 1997, p. 186.

[16] A. Nowicki, in Proceedings of IX Max Born Symposium New Symmetries in the Theories of Fundamental Interactions, Polish Scientific Publishers, (1997), p. 43 math.QA/9803064.

[17] P. Maślanka, J. Math. Phys. 34, 6025 (1993)

[18] J. Lukierski, Proceedings of Quantum Group Symposium at Group 21, (July 1996, Goslar) Eds. H.-D. Doebner and V.K. Dobrev, Heron Press, Sofia, 1997, p. 173.

[19] J. Lukierski, H. Ruegg and W.J. Zakrzewski, Ann. Phys. 243, 90 (1995).

[20] J. Lukierski, Proceedings of the III International Workshop on Classical and Quantum Integrable Systems, JINR (1998), hep-th/9812063].

[21] A. Kempf, G. Mangano, R. Mann, Phys. Rev. D52, 1108 (1995) hep-th/9412167]; A. Kempf, G. Mangano, Phys. Rev. D55, 1108 (1997) hep-th/9612084.

[22] M.R. Douglas and N.A. Nekrasov, Rev. Mod. Phys. 73, 977 (2002) [hep/th-0106048]

[23] H.S. Snyder, Phys. Rev. 71, 68 (1947). 